\title{
Die Hoggar-Tuareg im wirtschafts- und sozialgeographischen Umbruch
}

Anläßlich einer Expedition zur Beobachtung der totalen Sonnenfinsternis 1974 in die südalgerische Sahara hatte der Verfasser Gelegenheit, etliche Kontakte zu den Hoggar-Tuaregs anzuknüpfen und dabei wertvolle Einblicke in gegenwärtig sich abzeichnende kulturgeographische Veränderungen zu erlangen.

Über Lebensart und Wirtschaftsverhältnisse der Tuareg vor diesen Wandlungen bietet die Literatur hinreichende Informationen (1). Es sei deshalb nur mit einigen Sätzen darauf hingewiesen: "Schon seit Jahrhunderten bis kurz nach dem 2. Weltkrieg haben die Hoggar-Tuareg vorwiegend Kamele für die Kamelkarawanen und Kameltreiber sowie Karawanenführer gestellt. Dadurch fanden die Männer Arbeit und ihre Tiere aus der Kamelzucht guten Absatz. Ihre Haupteinnahmequelle war somit Tierzucht und Karawane. Die Kamelzucht wiederum bedingte einen Nomadismus, der sich - klimatisch gesteuert - in Jahrhunderten eingespielt hatte. Das Wohnzelt war daher leicht und mobil, für Ackerbau fand man nur in den allerseltensten Fällen Zeit, denn man war ja kaum länger als vier bis fünf Wochen am selben Ort. «Die französische Kolonisation brachte in den letzten 120 Jahren entscheidende Umwälzungen in die Sahara. Als bedeutendste Leistungen seien Tiefbohrungen nach Wasser, welche die Förderleistungen der früheren Brunnen um das Zehn- bis Hundertfache übertrafen, ErschlieBung von Erdöl- und Erdgasvorkommen und vor allem der moderne Straßenbau erwähnt.

Bald nach dem 2 . Weltkrieg wurden viele der einstigen Pisten zu breiten, teilweise asphaltierten Autostraßen ausgebaut. So konnten wir 1973 auf einer guten, sechs Meter breiten Asphaltstraße bereits bis Ain Salah gelangen, und bis 1975 soll der Belag durchgehend bis Tamanrasset im Hoggar reichen. Auf Strecken, die noch vor 20 Jahren nur mit Kamelen zu bewältigen waren, verkehren heute Lastwagen und sogar gewöhnliche Personenautos. In Sandgebieten allerdings bilden sich bei Stürmen häufig Verwehungen von gelegentlich einigen Metern Höhe, und es dauert dann oft Tage, bis der staatliche Sandräumungsdienst die blockierten Straßen mit Traxfahrzeugen wieder freigelegt hat.

Diese Entwicklung im Straßenbau setzte die Karawanen auf Kamelbasis außer Konkurrenz. Schon 1956 war die letzte Trans-Sahara-Karawane in Südtunesien eingetroffen. Seither sind die langsamen, an Tieren und Personal sehr aufwendigen Karawanen wirtschaftlich unrationell geworden. Die Traglast eines Kamels betrug 80 bis 150 Kilogramm. Für je zehn Lasttiere waren zwei bis drei Begleiter erforderlich, und die Tagesleistung der Karawane erreichte höchstens 25 bis 30 Kilometer. Für die Durchquerung der Sahara von Agades nach Tozeur wurden so 100 bis 120 Tage benötigt. Demgegenüber befördert ein Fernlaster, wie sie heute zu Hunderten die Wüste befahren, fünf bis acht Tonnen Nutzlast über Distanzen von 200 Kilometern und mehr im Tag. Dieser Vergleich mag erklären, weshalb das Kamel dem Lastwagen weichen mußte. Mag man den Karawanen mit ihrem Traditionalismus aus romantischen oder ethnographischen Gründen noch so sehr nachtrauern, genügte doch das Kamel den veränderten wirtschaftlichen Anforderungen nicht mehr. Es wird nach meinen Beobachtungen heute lediglich noch für privaten Gebrauch, als Reittier für Polizei und als Fleisch-, Milch-, Woll- sowie Lederlieferant verwendet.

Mit dem Karawanenverkehr verloren die Tuareg ihre wichtigste Einnahmequelle. Ihre Kamele fanden keinen Absatz mehr, die Möglichkeit, sie an Karawanen zu vermieten, fiel dahin, und Karawanenführer waren überflüssig geworden. Dies hatte eine beträchtliche Schrumpfung des Kamelbestandes zur Folge. Die Tierhaltung erfuhr tiefgreifende Veränderungen. Nach meinen Feststellungen werden heute viele Ziegen (schwarze und schwarz-braune Gebirgsziegen) und Schafe gehalten, auch scheint die Rinderzucht an Bedeutung zu gewinnen. So beobachtete ich in Amsel,

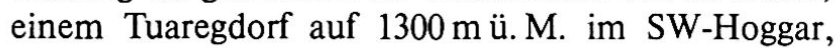
auf einer Stoppelweide (Maisfeld) ungefähr hundert Ziegen, zehn Schafe, zwei Rinder und zwei Esel. Dies war der Viehbesitz einer der hier noch häufig anzutreffenden Großfamilien, bei denen zwei bis drei Generationen in ein und derselben Behausung wohnen (Großeltern, Eltern, Kinder sowie Onkels und Tanten). Der Wegfall der Kamelzucht bewirkte nachhaltige Veränderungen in der Lebensweise der Tuareg. Viele haben schon das Nomadenzelt mit Lehm- oder Strohhäusern vertauscht. Die Verkleinerung der Herden entband die Hirten von der Notwendigkeit großer Wanderungen nach immer wieder neuen Futterplätzen. Es erfolgte der Übergang zum Halbnomadismus oder bereits zur Seßhaftigkeit. Soweit die kleiner gewordenen Herden noch Wanderungen erfordern,

Dr. Georg Jung-Blum, Hanfländerweg 20,8880 Walenstadt 
nimmt nur noch ein Teil der Familie, meist der Vater oder der Sohn, daran teil. Hält sich das Vieh aber in Reichweite der Dorfsiedlung auf, werden vorwiegend Frauen und Kinder für das Hüten eingesetzt.

Die Abkehr vom Vollnomadismus ermöglicht die Aufnahme von Bewässerungsfeldbau. Solcher ließ sich in mehreren Dörfern beobachten. Meist liegt ein geschlossenes Bewässerungsareal von zwei bis drei Hektaren außerhalb der Siedlung. Das Wasser wird gemeinsam hergeleitet und genutzt. In Amsel fand ich im Juli neben Dattelpalmen und Feigenbäumen folgende Anbaugewächse: Mais, Gurken, Wasser- und Zuckermelonen, Zucchetti, Tomaten und Auberginen. Natürlich wird dieser Acker- und Gemüsebau durch das nur begrenzt zur Verfügung stehende Wasser stark eingeschränkt. Düngung unterbleibt weitgehend, zumal wegen des Mangels an Holz getrockneter Mist (vor allem Kamelmist) als Brennmaterial zu dienen hat. Leider war es nicht möglich, Angaben über die geernteten Mengen zu erhalten. Waagen sind keine vorhanden, und da die Tuareg noch vielfach Analphabeten sind, darf kein Sinn für Buchhaltung und Statistik vorausgesetzt werden. Nach Schätzungen aber bedeutet der neue Bewässerungsfeldbau eine wesentliche Bereicherung der bisherigen Ernährung, und bereits zeichnet sich auch eine Marktorientierung ab. In Tamanrasset ziehen zeitweise Gemüsefahrer auf den lokalen Markt, um dort bescheidene Überschüsse abzusetzen, und Touristenzentren werden beispielsweise mit Zuckermelonen beliefert.

So erfolgversprechend diese neue Entwicklung zunächst auch erscheinen mag, wird sie doch von neuerlich beobachteten Klimaverschlechterungen wieder gefährdet. In Tamanrasset sind während der letzten sieben Jahre zu wenig bis überhaupt keine Niederschläge gefallen. Offenbar handelt es sich dabei um eine Randerscheinung der Niederschlagsdefizite in der südlich gelegenen Sahelzone. Für Tamanrasset gibt J. DUBIEF (2) folgende Klimawerte an:

$\begin{array}{ll}\text { Jährliche Niederschlagsmenge } & 30 \mathrm{~mm} \\ \text { Schwankung } & 5 \mathrm{~mm} \text { bis } 155 \mathrm{~mm} \\ \text { Regenhäufigkeit } & 6,9 \mathrm{Tage} / \mathrm{Jahr}\end{array}$

Temperaturmittel:

Juli $28^{\circ}$, Januar $11^{\circ}$, Jahresmittel $20^{\circ}$

Ariditätsindex $\quad 0,11$

Daraus geht hervor, daß Wasser äußerst spärlich zur Verfügung steht. In Tamanrasset (ca. 2000 Ew.) mußte in den letzten Jahren der Trinkwasserverbrauch durch drastische Sparmaßnahmen empfindlich eingeschränkt werden. So waren die Brunnen morgens und abends nur je eine Stunde in Betrieb. Daß unter solchen Umständen die sanitären Installationen in modern ausgestatteten Hotels weitgehend ihrer Funktion nicht zu genügen vermögen, sei nur nebenbei vermerkt.

Der Übergang zur seßhaften Lebensweise führt zu grundlegenden Veränderungen in der Siedlungsstruktur. Das leicht transportierbare Zelt, einst das bezeichnendste Siedlungselement, verschwindet allmählich. Es scheint gegenwärtig nur noch Familien als Behausung zu dienen, die entweder keine Mittel für den Hausbau besitzen oder sich aus einer konservativen Haltung heraus nicht von der traditionellen Wohnweise trennen wollen. Gelegentlich mögen es auch alte Leute sein, die für den Rest ihres Lebens einen Neubau nicht mehr als sinnvoll empfinden.

In einer kleinen Tuaregsiedlung östlich von Tamanrasset, deren Grundriß in Fig. 1 festgehalten ist, waren im Juli 1973 unter den 39 vorhandenen Bauten 24 Lehmhäuser, 14 Zelte und 1 Strohhaus zu zählen. Nach meinen Beobachtungen scheint gegenwärtig dem Lehmhaus gewissermaßen ein höherer Sozialstatus zuzukommen.

\section{Das Lehmhaus (Abb. 1)}

Das Lehmhaus wird von einer Familie, bzw. einer Sippe, in eigener Arbeit erstellt. Von nahe gelegenen Lehmvorkommen, wie sie vielfach in Wadis zu finden sind, stammt das Baumaterial. Die Ziegel haben Ausmaße von $20 \times 18 \times 12 \mathrm{~cm}$ oder gelegentlich auch etwas mehr. Sie werden nicht gebrannt, sondern nur an der Sonne getrocknet. Das Aufmauern geschieht ohne Mörtel. Man befeuchtet nur die Flächen der Ziegel, welche versetzt aufeinander zu liegen kommen. $\mathrm{Da}$ der Lehm oft etwas eisenschüssig ist, erhalten die Mauern eine rötliche Farbe. Will man etwas mehr Mühe und Arbeit investieren, werden die Außenwände mit Lehm verputzt und hernach mit Strichtechnik verziert. Oft aber verzichtet man darauf. Für die Bedachung gelangt heute meistens Wellblech zur Verwendung. Da die Mauern das Dach umkragen, bleibt die Bedeckung weitgehend verborgen und stört darum vom ästhetischen Standpunkt aus nicht. 
Die Türen des Hauses bestehen aus Holz oder Blech. Je nach der Größe der Familie wird das Innere in zwei bis drei Räume unterteilt. Aus klimatischen Gründen fehlen meist die Fenster. Die Kammern dienen in erster Linie als Schlaf- und Vorratsräume. Das tägliche Leben spielt sich eher im Vorhof oder draußen vor dem Hause ab.

Arbeiten, Kochen und Essen beschränken sich auf die früheren Vormittagsstunden. Wenn gegen neun Uhr die Hitze des Tages sich bemerkbar zu machen beginnt, flüchten die Bewohner in den Schatten des Vorhofes, der teilweise mit Palmblättern oder Maisstroh überdeckt ist, oder in die inneren Räume. Sie legen sich zum Schlafen nieder oder verbringen beim würzigen Pfefferminztee die Zeit mit Gesprächen, Gesang und Spiel. Erst gegen fünf Uhr nachmittags erwacht das Leben erneut und verstummt dann gegen Mitternacht oder noch später.

Das Mobiliar der Tuareg erscheint äußerst dürftig. Stühle und Tische sind noch weitgehend unbekannt. Die Leute setzen sich auf dem Boden zusammen, der bei sozial besser Gestellten mit Strohmatten oder Wolltüchern belegt wird, bei den Ärmsten aber nackt bleibt. Als Feuerstelle dient immer noch die Lehmgrube in der Mitte des Wohnraums. Der Rauch zieht durch die Türe oder eine Dachöffnung ab. Mühsam muß das Brennholz in den Wadis zusammengesucht werden und ist darum sehr kostbar. Emaillierte Eisengefäße und $\mathrm{ab}$ und zu auch schon Aluminiumpfannen dienen als Kochgeschirr. Aber auch von Touristen weggeworfene Konservenbüchsen oder leere Ölkanister, die irgendwo aufgefunden worden sind, finden etwa den Weg in die Küchen.

Im Gegensatz zum ärmlichen Hausrat überrascht immer wieder der reiche Silberschmuck, den die meisten der Frauen tragen. Auch die Männer pflegen sich oft zu schmücken. Hier kommt noch augenfällig zum Ausdruck, daß die Tuareg ihre Ersparnisse sozusagen ausschließlich auf diese Weise in Sachwerten anzulegen suchen. Die Tuaregfrauen werden deshalb gewissermaßen zu «wandelnden Sparkassen».

\section{Das Strohhaus (Abb. 2)}

Das Strohhaus stellt vielfach den Übergangstypus vom Zelt zum Lehmhaus dar. Als Tragkonstruktion birgt es ein Astgabelwerk, das an einen Dachstuhl erinnert. Wände und Dach bestehen aus Palmfasermatten. Da es nur selten oder überhaupt nie regnet, verursacht die Abdichtung keinerlei Problem. Das Haus hat vorwiegend Wind- und Sonnenschutz zu gewähren. Wie beim Lehmhaus liegt die Feuerstelle etwas seitlich und besteht aus einer etwa schuhtiefen Grube. Die Grundfläche der Behausung beträgt 50 bis $80 \mathrm{~m}^{2}$. Etwa der fünfte Teil wird von der Küche eingenommen, ein weiterer Fünftel dient der Aufbewahrung des Hausrates und der Rest steht für Wohnen und Schlafen zur Verfügung. Die lichte Höhe der Räume mißt durchschnittlich $180 \mathrm{~cm}$.

\section{Das Zelt (Abb. 3)}

Das Zelt weist zur Hauptsache Trapezform auf. Giebelform ist seltener vertreten. In der Regel sind die zwei offenen Seiten nach Norden und Süden orientiert. Das Trapezzelt ist so niedrig, daß die Bewohner darin nicht aufrecht stehen können. Tamariskenholz liefert die meist krummen Zeltstangen. Auf senkrecht in den Boden gerammten Astgabeln ruhen mehr oder weniger parallel verlegte Horizontalhölzer. Darüber sind Zeltplanen aus Baumwolle, seltener aus Kamelhaarfilz oder Ziegenleder verspannt. Kleine Holzpfähle dienen als Zeltpflöcke. Ist der Boden aber zu hart, oder besteht er aus anstehendem Fels, werden die Schnüre mit großen Steinen beschwert. Die Grundfläche solcher Zelte beträgt ungefähr 20 bis $30 \mathrm{~m}^{2}$.

Zum Abschluß sei noch auf einige sozialgeographischen Beobachtungen hingewiesen:

Die Verarztung der Bevölkerung läßt sehr zu wünschen übrig. Nur in Tamanrasset praktiziert ein Arzt, der insgesamt 5000 Menschen auf einer Wohnfläche von der Größe Ungarns zu betreuen hätte. Die Siedlungen liegen meistens 50 Kilometer oder mehr auseinander, das heißt zwei Tage Kamelritt. So verunmöglichen schon die weiten Anmarschwege für die Bewohner den Gang zum Mediziner. Weitere Hindernisse sind Geldmangel und oft noch tief verwurzelte Vorurteile. Beinahe in jeder Behausung, die wir besuchen konnten, trafen wir auf Patienten, die - meist nur in eine Decke gewickelt - auf dem Boden lagen. Moderne Medikamente sind einzig in Form von Schmerzlinderungsmitteln bekannt und stehen hoch im Kurs. Wir wurden fast bei jeder Begegnung in gebrochenem Französisch darnach gefragt («Pillule - mal tête - mal estomac»). Einmal ließen wir einen Targi aus einem 
Fig. 1: Grundriß einer Tuareg-Siedlung.

Fig. 2: Das Lehmhaus der Tuareg und seine Einteilung.
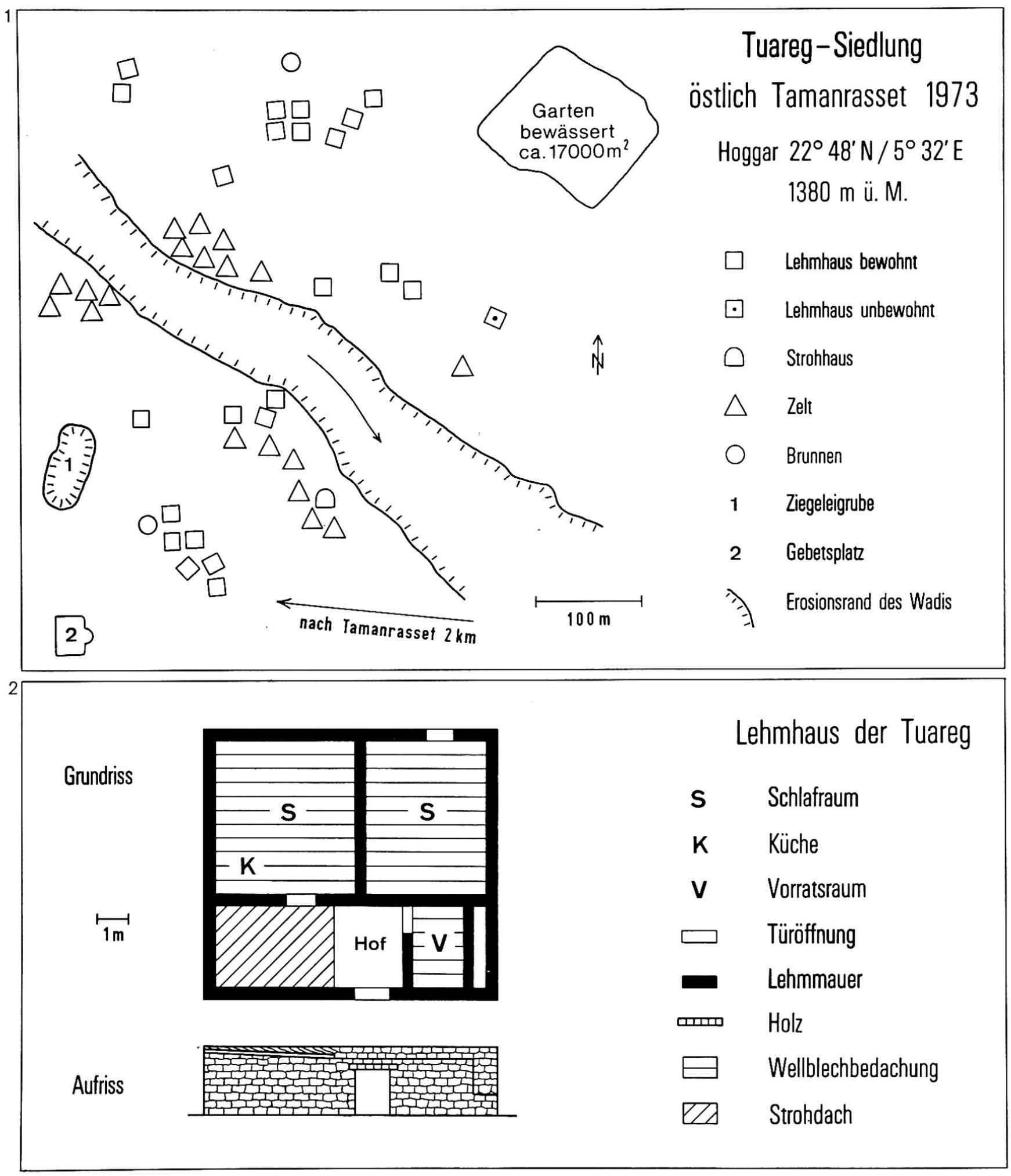
Abb.1: Lehmbehausung der Hoggar-Tuareg, die Türe ist aus Holz, das Mauerwerk besteht aus ungebrannten Lehmziegeln. Vor dem Haus steht eine einfache Bettstelle (Dorf östlich Tamanrasset).

Abb. 2: Strohhaus der Hoggar-Tuareg. Das Moped dient dem Besitzer als Transportmittel, um für das ganze Dorf Einkäufe zu tätigen. Das Haus steht in Amsel (SW-Hoggar).

Abb. 3: Tuareg-Zelte im Dorf östlich von Tamanrasset. Rechts sind Lehmhäuser zu sehen, im Hintergrund vulkanische Basaltstöcke des Atakor.

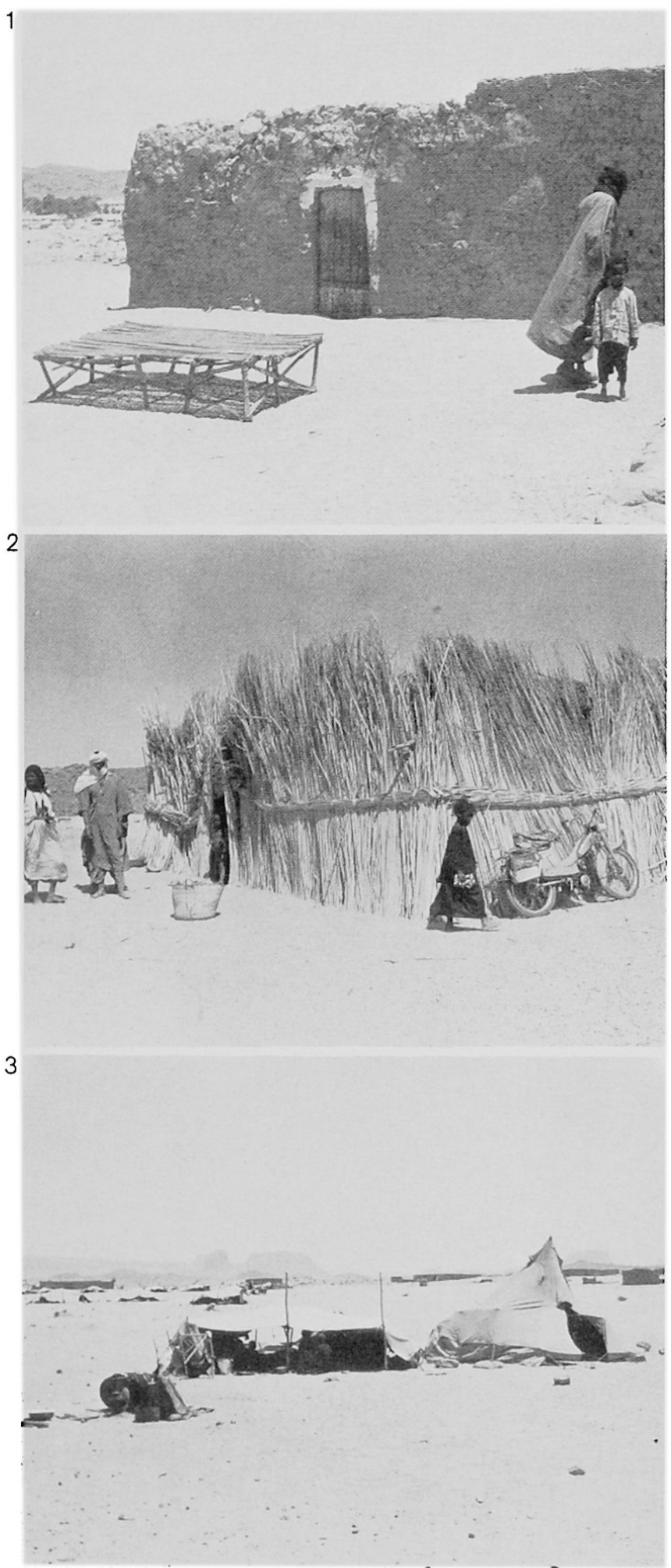

Abb. 4: Ziegen auf einer Weide in einem Wadi. Das Hüten ist oft Aufgabe der Frauen oder Kinder. Im Hintergrund sind Wollsackverwitterungsformen im Granit zu sehen.

Abb. 5: Bewässerungsfeldbau der Hoggar-Tuareg im Dorf Amsel auf 1300 m Meereshöhe. Nebst Dattelpalmen wachsen Feigen und Gemüse. (Bild im Juli aufgenommen.)

Abb. 6: Hoggar-Tuaregfamilie beim Singen. Während Sohn und Kleinkinder klatschen und einen fixen Ton summen, tremuliert die Mutter in einer Diskantmelodie in freien improvisierten Rhythmen. Ein leerer Benzinkanister dient als Trommel.
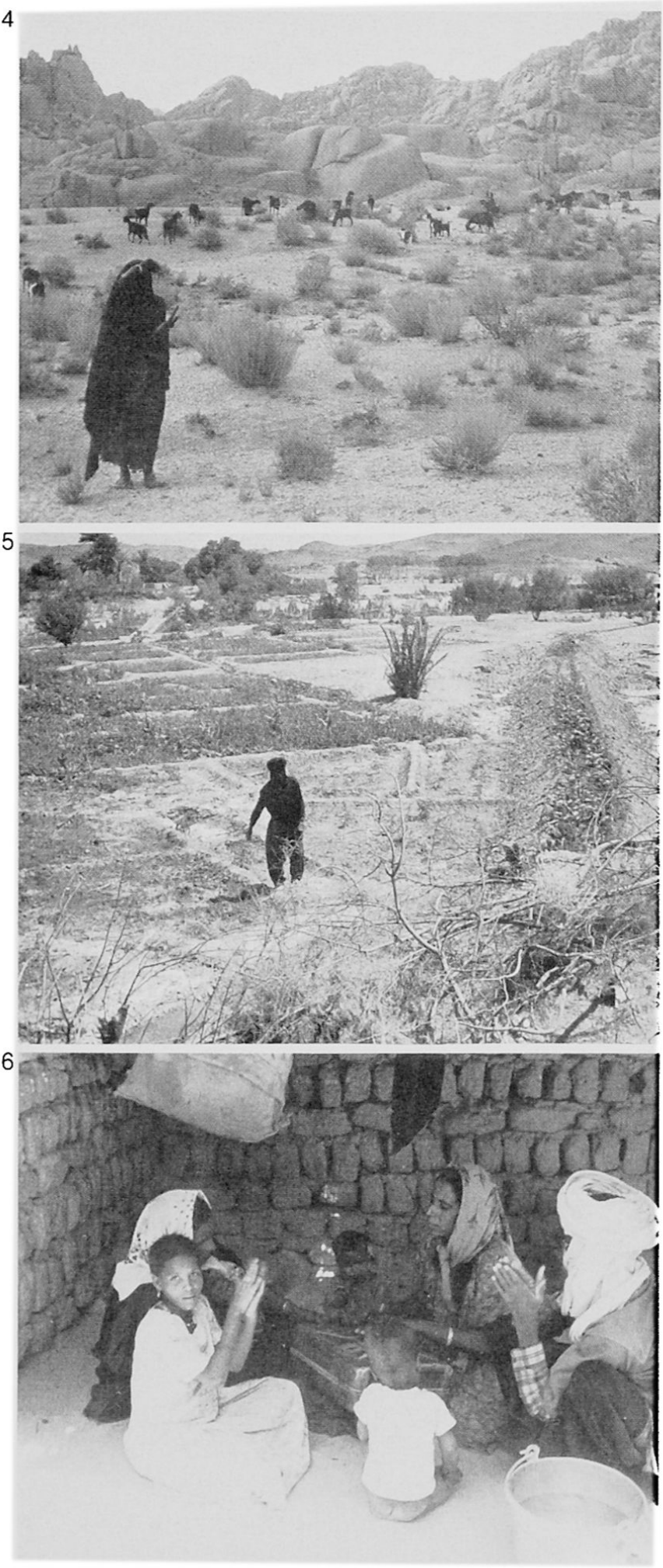
abgelegenen Dorf im Landrover nach Tamanrasset mitfahren, der für die ganze Dorfbevölkerung beim Arzt Medikamente zu besorgen hatte. Natürlich kann mangels Diagnose in den allerwenigsten Fällen zweckmäßig geholfen werden.

Unter der Arbeitslosigkeit als Folge der wirtschaftlichen Veränderungen haben vor allem die jungen Männer zu leiden. Zwar sind die Tuareg recht aufgeweckt und intelligent. Sprachen lernen sie rasch. Ein junger Mann, der schon etwas Französisch sprach, hatte sich bereits nach einer Woche Umgang mit uns einen deutschen Wortschatz von einigen Dutzend Wörtern angeeignet. Auch mit dem Auto verstehen sie bald umzugehen. Doch sind die Arbeitsplätze dünn gesät. Meist handelt es sich um solche im tertiären Sektor. So bleibt nur die Auswanderung nach den Oasen übrig. In Zelfana beispielsweise, $1000 \mathrm{~km}$ nördlich des Hoggar, trafen wir einen Targi aus Tamanrasset, der dort als Kellner arbeitet.

Groß ist immer noch - trotz verhältnismäßig hoher Kindersterblichkeit - der Kinderreichtum der Familien. Von den 400 Einwohnern eines Dorfes im Osten von Tamanrasset haben, wie wir feststellen konnten, 250 das Alter von 18 Jahren noch nicht erreicht. Vier bis zehn Kinder je Elternpaar dürfen als Regel gelten. Was uns erstaunte, ist der Versuch der algerischen Verwaltung, die Tuareg mittels Ausweispapieren unter Kontrolle zu bringen. Jeder besitzt einen amtlichen Ausweis mit Photographie, und es kann geschehen, $\mathrm{da} ß$ die Polizei in größeren Ortschaften die Legitimation überprüft. Allerdings erfolgt die Erfassung der Bevölkerung erst im Erwachsenenalter.

Noch immer tragen die älteren Männer den Gesichtsschleier, und zwar offensichtlich mit Stolz. Die jüngeren haben ihn wohl vom Vater beim Eintritt in die Pubertät erhalten, binden ihn jedoch nur noch selten oder überhaupt nicht mehr vor. Kaftan und Wollhose sowie der Turban sind allerdings noch durchwegs in Gebrauch. Man beobachtet aber immer wieder, daß sich europäische Kleidungsstücke dazugesellen, die in keiner Weise zur traditionellen Gewandung passen. Bei vielen beobachtet man sogar eine Erhöhung des Statusgefühls, sobald sie Träger eines europäischen Kleidungsstückes sind, das sie in der Regel durch ein Tauschgeschäft mit Touristen erworben haben.

Oft sind es auch technische Geräte, welche den Status des einzelnen heben. So zählt der Besitz einer Uhr oder eines Transistorenradios zu den bedeutendsten
Wünschen eines Targi. Im Dorf Amsel, rund $50 \mathrm{~km}$ südlich von Tamanrasset, trafen wir auf ein Moped. Dessen Besitzer fährt damit öfters nach Tamanrasset und besorgt für das ganze Dorf Einkäufe, insbesondere von Medikamenten und Konsumgütern. Dafür erhält er einen «Fuhrlohn», mit dem er offenbar sein Fahrzeug amortisiert. Er gilt dank des Mopeds als höchst angesehene Person und wird, vor allem von der Dorfjugend, entsprechend verehrt und auch beneidet. Anderseits fehlen diesem Dorf jegliche sanitäre Einrichtungen und modernen Werkzeuge sowie die elektrische Energie. Schon ein Taschenmesser löst dort Erstaunen aus. Die Wasserversorgung erfolgt mittels Brunnen, aber noch lange nicht jede Haushaltung verfügt über einen solchen.

Trotz ihrer scheinbaren Armut machen die Tuareg durchwegs einen recht zufriedenen Eindruck. Meiner Ansicht nach könnte ihr Leben durch verbesserte medizinische Betreuung wesentlich erleichtert werden. Durch die Verteilung von zweckmäßigeren Werkzeugen und Anleitung zu verbesserten Anbaumethoden unter Benützung von Dünger ließen sich die Erträge ihres Acker- und Gemüsebaues erheblich steigern. Doch scheint es mir verfehlt, wenn man diese Menschen mit allen zivilisatorischen Errungenschaften vertraut $\mathrm{zu}$ machen versucht. 'Vermutlich würden sie dabei mit der Zeit recht unzufrieden, und zudem liefen sie Gefahr, eine ähnlich umweltschädigende Entwicklung durchzumachen wie europäische Völker. Mögen sie davor bewahrt bleiben!

\section{Benützte Literatur:}

(1) LHOTE H., Les Tourags du Hoggar, Paris, 1955

(2) DUBIEF J., Die Sahara, eine Klimawüste, in «Sahara und ihre Randgebiete» von H. SCHIFFERS, München, 1971

(3) NORWICH J. J., Sahara, Safari Verlag, Berlin, 1967 